\title{
Optical Study of Laser Ablation Plasma Irradiation for the Bacteria Sterilization using Metal Oxide Target
}

\author{
H. Kawasaki, T. Ohshima, Y. Yagyu, T. Ihara, M. Yamauchi, Y. Suda \\ National Institute of Technology, Sasebo College, 1-1 Okishin, Sasebo, 857-1193, Japan \\ Fax: 81-956-34-8409, e-mail:h-kawasa@sasebo.ac.jp
}

\begin{abstract}
PLA plasma sterilization of yeast fungus and spore-forming bacteria was performed using $\mathrm{Ti}$ and $\mathrm{TiO}_{2}$ target. The experimental results suggested that both the yeast fungus and spore-forming bacteria could be sterilized by plasma irradiation generated by pulsed laser ablation method. The effect using pure Ti target slightly stronger than that of the $\mathrm{TiO}_{2}$ target. This results suggests that the main sterilization factors were considered to be high energy metal atoms and/or ions in this system.
\end{abstract}

Key words: plasma sterilization, pulsed laser ablation, plasma process

\section{INTRODUCTION}

Recently, plasma sterilization has been used for various fields, such as medicine, pharmacy, agriculture, etc. $[1,2]$. An advantage of the plasma sterilization method is its potential, under appropriate conditions, to be achieved at relatively low temperatures, thereby preserving the integrity of polymer-based instruments, which cannot be subjected to autoclaves and ovens. Therefore, plasma sterilization is safe, for both operator and patient. As the results of these advantages, plasma sterilization has attracted much attention in recent years. For example, Hayashi et al. developed a high-speed method for sterilizing PET bottles using a microwave plasma torch [3-5]. Yagyu et al. suggest that glow discharge plasma irradiation for some kinds of plants, such as Bean sprouts, increased their growth rate[6]. In particular, atmospheric plasmas, such as plasma jets and surface discharges, have been used as typical sources for plasma sterilization. However, the effects of atmospheric plasma on several kinds of bacteria are not strong, and long-term sterilization damages the operator, patient, and substrate that is placed on the sterilization subject [7-10].

The pulsed laser deposition (PLD) method is widely used technique for the deposition of thin films because of its numerous advantages, such as a simple system setup, low temperature, and high instantaneous deposition rates. We have prepared functional thin films under various deposition conditions using the PLD method, and all of them showed high quality, including high crystallinity and hardness without substrate heating [11-14]. In general, it is considered that the degree of ionization at the very close to the target during the early phase of pulsed laser ablation (PLA) plasma should be very high, and the plasma area may be very reactive. Therefore, it is believed that PLD plasma will be effective for types of bacteria sterilization that are difficult by other methods. We have been studied about plasma sterilization using PLA derived plasma. Previously, PLA plasma, generated from Ag bulk target, was used for the yeast fungus and spore-forming bacteria sterilization. Experimental results suggest that the PLA plasma was very effective to those bacteria sterilization, however the mechanisms and special effects of the PLA sterilization cannot be investigated. Especially, it is not clearly solved that what is the most important effective species in the PLA plasma sterilization[15].

In this experiment, yeast fungus and spore-forming bacteria were used as the subjects of sterilization, and $\mathrm{Ti}$ and $\mathrm{TiO}_{2}$ bulk was used as the PLD target. In addition, the properties of the processing plasma were studied. The emission spectra of the PLA-derived plasma were measured with a monochromator. Based on these results, the mechanisms of plasma sterilization using highly ionized pulsed plasma were explored.

\section{EXPERIMENTAL}

The plasma sterilization system using PLA derived plasma is normal PLD system to prepare functional thin films as shown in previous work[18]. The spherical chamber was composed of stainless steel $400 \mathrm{~mm}$ in diameter. In this experiment, bulk $\mathrm{Ti}$ and $\mathrm{TiO}_{2}$ were used for the target. A pulsed Nd:YAG laser (Spectra-Physics Quanta-Ray PRO-230-10; wavelength $532 \mathrm{~nm}$, pulse duration 1 - $2 \mathrm{~ns}$, maximum output energy $650 \mathrm{~mJ}$ ) was used to irradiate the targets. The radiated area on the targets was maintained at $0.32 \mathrm{~cm}^{2}$. The laser fluences used were $1-10 \mathrm{~J} / \mathrm{cm}^{2}$. The chamber was evacuated to $10 \mathrm{~Pa}$ using an oil free scroll pump, and then reduced to a base pressure under $1 \times 10^{-3} \mathrm{~Pa}$ using a turbomolecular pump. A deposition total pressure of 10 
$\mathrm{Pa}$ was produced using a mixture of pure $\mathrm{O}_{2}$ gas at a flow rate of $10 \mathrm{sccm}$.

Yeast fungus and spore-forming bacteria were used as the subjects of sterilization in this experiment. These samples were placed on a Si substrate, which was used as the PLD substrate holder. A plasma plume, which was generated by PLA on the $\mathrm{Ti}$ and $\mathrm{TiO}_{2}$ bulk targets ( $>$ 99.5\%, Koujundo Chem. Lab. Co.,Ltd.), was irradiated to the Si substrate containing the sterilization subjects. After plasma irradiation, the effects of plasma sterilization were judged by counting the number of colony forming units (AOAC International, Sanita-kun) and liquid medium (ProPACT). The emission spectrum of the PLA derived plasma was measured with a monochromator (Hamamatu photonics PMA-50) using a 20-ns fast gate pulse.

\section{Results and Discussion}

\subsection{Emission spectra of PLA derived plasma}

Figure 1 shows the typical optical emission spectra at the center position of the plasma plume produced by ablating $\mathrm{Ti}$ and $\mathrm{TiO}_{2}$ targets. As the results, several strong $\mathrm{Ti}$ atoms (Ti $\mathrm{I}, \lambda=307,335$, $390,445,453,626 \mathrm{~nm}$, etc) and Ti ions (Ti II, $\lambda=323$, $335,375 \mathrm{~nm}$ etc) and $\mathrm{O}$ atoms (O I, $\lambda=437 \mathrm{~nm}$, $777 \mathrm{~nm}$, etc) peaks were observed in the plasma plume. This result suggest that those species exist in the plasma plume.

Figure 2 shows the temporal evolutions of optical emission spectra in the plasma plume using $\mathrm{TiO}_{2}$ target as functions of measurement position. In those Figs. 2(a), 2(b), 2(c) shows 1, 3, $10 \mathrm{~mm}$ from the target's surface, respectively. As those results, intensities of the emission were strong in the early phase of plasma plume

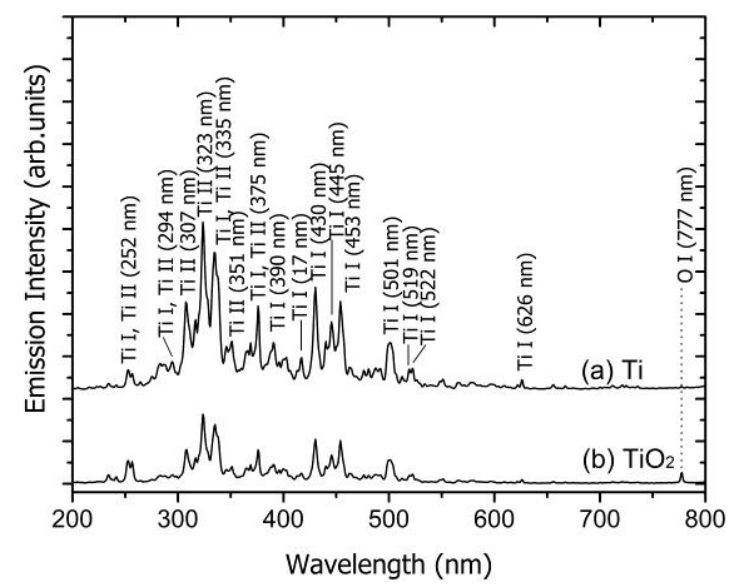

Fig. 1 Typical optical emission spectra at the center position of the plasma plume produced by ablating $\mathrm{Ti}$ and $\mathrm{TiO}_{2}$ targets. generation at close position from the target. At the far place from the target, $\mathrm{Ti}$ ions and $\mathrm{O}$ atoms emission disappeared, and weak Ti atom emission can be observed. In this experiment emissions of $\mathrm{O}_{2}$ molecules $(530,560,600,640 \mathrm{~nm})$ cannot be observed clearly. We also measured the temporal evolutions of optical emission spectra in the plasma plume using $\mathrm{Ti}$ target as functions of measurement position, not shown here. The peak intensity of the spectra were different, but dependence of the distance from the target and delay time were almost same compared with that of $\mathrm{TiO}_{2}$ target. This result suggests that high energy $\mathrm{Ti}$ atoms and ions existed in the plasma plume, and that plasma sterilization can be performed by those species using the PLA

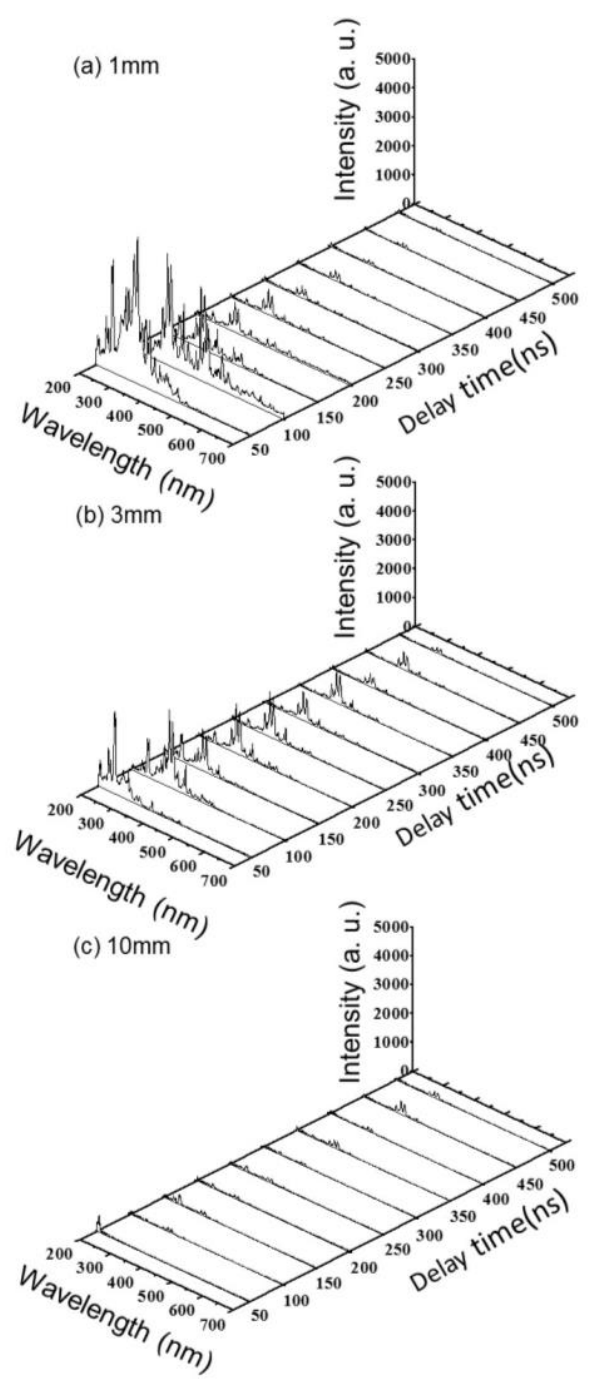

Fig. 2 Temporal evolutions of optical emission spectra using $\mathrm{TiO}_{2}$ target. 
derived plasma.

Figure 3 and 4 shows emission peak intensity of Ti atoms (Ti I, $430 \mathrm{~nm}$ ), Ti ions (Ti II, $323 \mathrm{~nm}$ ), and $\mathrm{O}$ atoms (O I, $777 \mathrm{~nm}$ ) using $\mathrm{Ti}$ and $\mathrm{TiO}_{2}$ target. As the results, peak intensity of the $\mathrm{Ti}$ I using Ti target was higher than that using $\mathrm{TiO}_{2}$ target, especially at closed position from the target. However, peak intensity of the $\mathrm{O}$ I atoms using $\mathrm{TiO}_{2}$ target was higher than that of the Ti target. Those results suggest that the high energy $\mathrm{Ti}$ atoms and ions densities in the plasma plume using $\mathrm{Ti}$ target were higher than that using $\mathrm{TiO}_{2}$ target. However, high energy $\mathrm{O}$ atoms densities in the plasma plume using $\mathrm{Ti}$ target were lower than that using $\mathrm{TiO}_{2}$ target in this experiment.
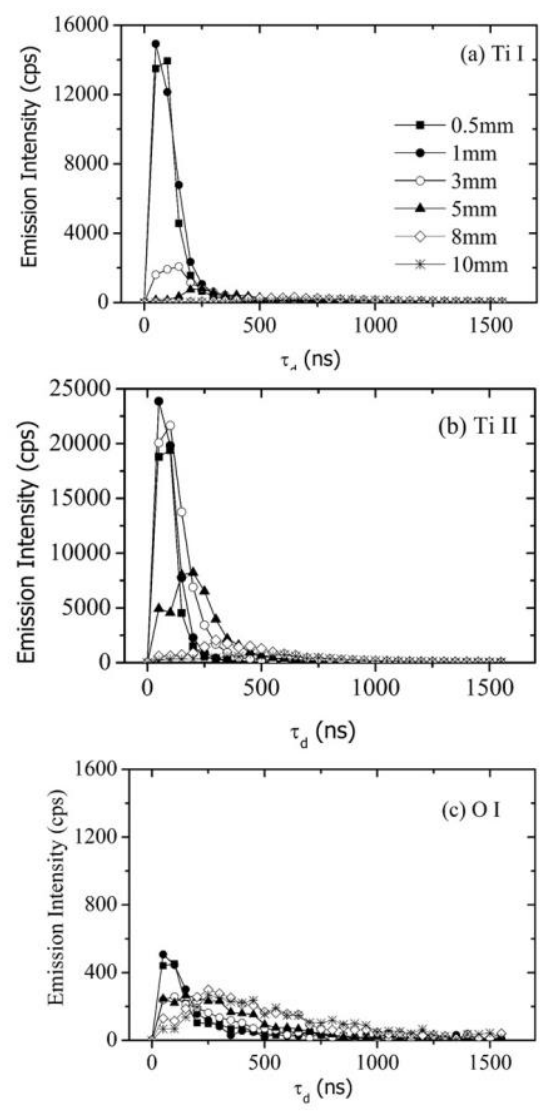

Fig. 3 Temporal evolution of emission peak intensity of Ti I, TiII and O I using Ti target.

3.2 Plasma sterilization of yeast fungus, viable bacteria count and spore-forming bacteria by the PLA method

Figure 5 and 6 show the effect of PLA sterilization for the viable bacteria count, yeast fungus and spore-forming bacteria as parameters of the target materials. In this experiment, the effects of plasma sterilization were judged by counting the number of colony forming units. At first, the number of colony forming units (control value: $\mathrm{Nv}$ ) without
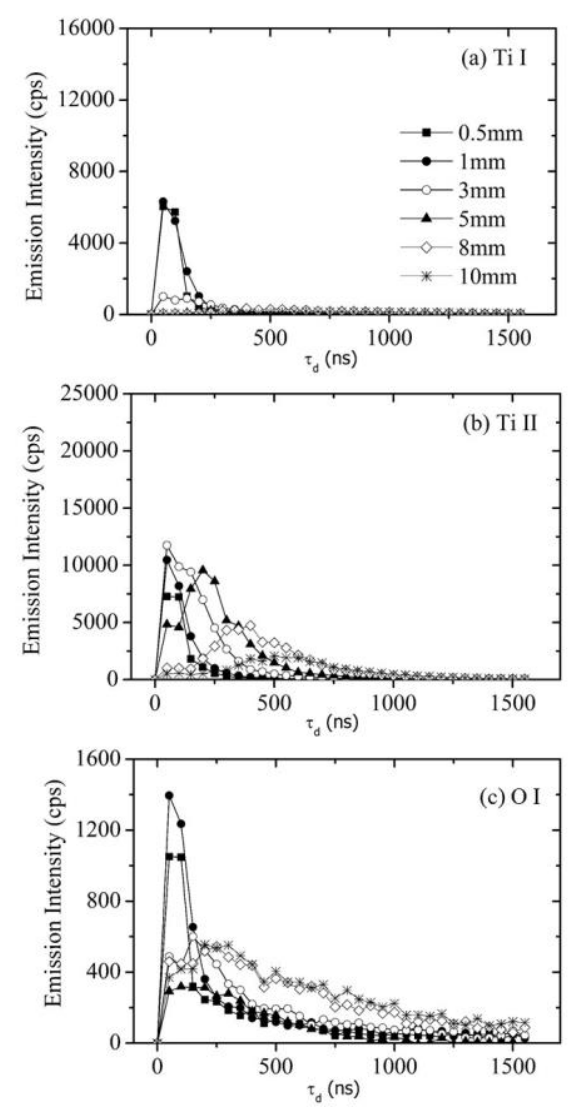

Fig. 4 Temporal evolution of emission peak intensity of Ti I, TiII and O I using $\mathrm{TiO}_{2}$ target.

PLA plasma irradiation was counted. After that PLA plasma irradiated to the substrate with the viable bacteria count. And then it standardizes with the Nv value.

To study the effects of plasma sterilization, spore-forming bacteria were cultivated in a liquid medium (ProPACT) after PLA plasma irradiation. Cultivation was performed at a temperature of $60{ }^{\circ} \mathrm{C}$ in a liquid medium for $24 \mathrm{~h}$. The color of

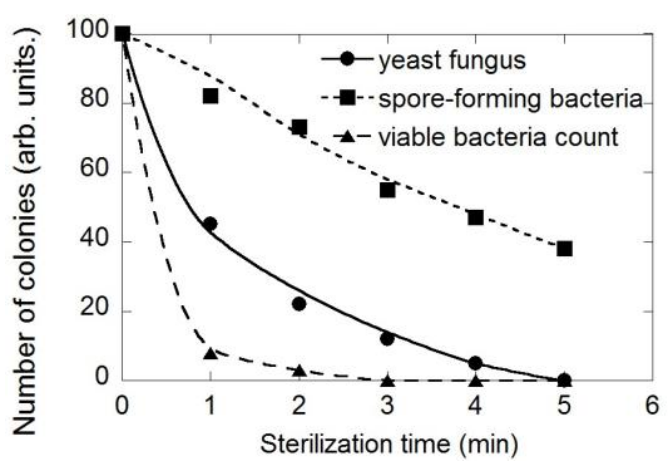

Fig. 5 Effects of PLA sterilization for the viable bacteria count, yeast fungus and spore-forming bacteria using Ti target. 
the liquid medium was changed from yellow to dark brawn if the spore-forming bacteria were sterilized. Therefore, sterilized effects were judged by laser light transmittance of the liquid.

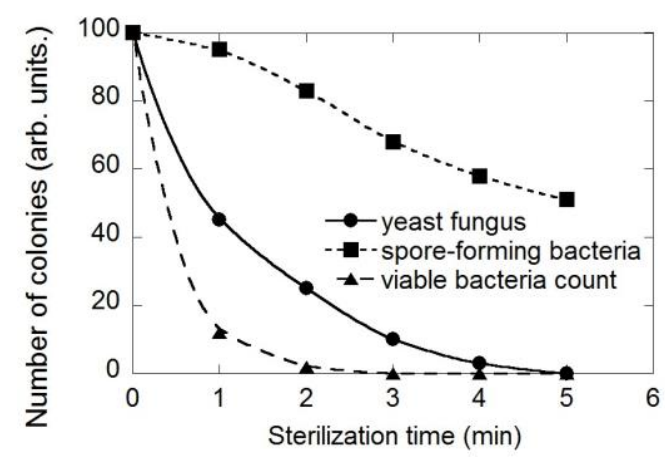

Fig. 6 Effects of PLA sterilization for the viable bacteria count, yeast fungus and spore-forming bacteria using $\mathrm{TiO}_{2}$ target.

As shown in these results viable bacteria count decreased by PLA plasma irradiation using $\mathrm{Ti}$ $\mathrm{TiO}_{2}$ targets. Dependence of the sterilizing effects on the target materials cannot be clearly observed. However, the effect using pure $\mathrm{Ti}$ target slightly stronger than that of the metal oxide target.

The mechanism of sterilization by PLA was examined based on the results mentioned above. The factors involved in sterilization by the PLA derived plasma were considered to be as follows: 1) thermal reaction, 2) high energy UV light: 3) various high energy atoms and molecules originates by gas, and 4) high energy metal atoms and ions that ablated from the target. In our experiments, the surface temperature after 5 min of PLA derived plasma irradiation was less than $80{ }^{\circ} \mathrm{C}$ as measured by radiation thermometers, not shown here. Thus, it is unlikely that the mechanism of the sterilization was direct heating of the subjects. Optical emission spectra suggest that intensity of the $\mathrm{O}_{2}$ molecules emission cannot be observed, and $\mathrm{O}$ atom emission was very weak. Therefore, the density of high energy $\mathrm{O}$ atoms and $\mathrm{O}_{2}$ molecules, which may have been generated from the gas existing in the chamber, was considered to be very low. The results suggest that $\mathrm{O}$ atoms and/or $\mathrm{O}_{2}$ molecules were not strongly effects for the bacteria sterilization in this system. The intensity of the UV light of the PLA derived plasma decreased rapidly with the delay time, and the intensity was almost zero. In addition, difference of the sterilization effect on the target material cannot be appeared clearly in this experiment. These results suggest that high energy Ti atoms and ions exist in the PLA derived plasma. In general, Ti atoms and ions have bactericidal effects, including active $\mathrm{O}_{2}$ generation by a catalyst reaction of $\mathrm{Ti}$ and the destructive effect of $\mathrm{Ti}$ invading bacteria cells. Based on these results, it is believed that the main sterilization factors are high energy metal atoms and/or ions in this system.

\section{CONCLUSION}

PLA plasma sterilization of yeast fungus and spore-forming bacteria was performed using $\mathrm{Ti}$ and $\mathrm{TiO}_{2}$ target. The experimental results suggested that both the yeast fungus and spore-forming bacteria could be sterilized by plasma irradiation generated by pulsed laser. The dependence of the effects of the sterilization on the target materials cannot be clearly observed. However, the effect using $\mathrm{Ti}$ target slightly stronger than that of the pure $\mathrm{TiO}_{2}$ target. Therefore, it is considered that high energy metal atoms and/or ions were strongly effects for the PLA plasma sterilization.

\section{References}

[1] M. Moisan, J. Barbeau, J. Pelletier. Le vide: Sci. Tech. Appl. 299, 15-28 (2001).

[2] M. Moisan, J. Barbeau, S. Moreau, J. Pelletier, M Tabrizian, L'H. Yahia. Int. J. Pharm. 226, 1-21 (2001).

[3] N. Hayashi, T. Nakashima, A. Yonesu. IEEE Trans. on Plasma Science, 39, 2976 (2011).

[4] N. Hayashi, A. Nakahigashi, M. Goto, S. Kitazaki, K. Koga, M. Shiratani. Jpn. J. Appl. Phys, 50, 08JF04 (2011).

[5] S. Kitazaki, A. Tanaka, N. Hayashi, VACUUM,110,217, (2014).

[6] Y. Yagyu, A. Hikida, N. Hayashi, H. Kawasaki, T. Ohshima, Y. Suda, Trans. Mat. Res. Soc. Jpn, 35[1] 119 (2010)

[7] H. Yasuda, T. Miura, H. Kurita, K. Takashima, and A. Mizuno. Plasma Proc. Polymers 7, 301 (2010)

[8] S. Ikawa, K. Kitano, S. Hamaguchi. Plasma Proc. Polym. 7(1), 33(2010)

[9] I. Motrescu, A. Ogino, S. Tanaka, T. Fujiwara, S. Kodani, H. Kawagishi, G. Popa, M. Nagatsu. Jpn. J. Appl. Phys. 50, 08JF07 (2011).

[10] H. Shintani, A. Sakudo, P. Burke, G. Mcdonnell. Exp Ther Med. 1, 731 (2010).

[11] H. Kawasaki, T. Ohshima, K. Arafune, Y. Yagyu, Y. Suda. Jpn. J. Appl. Phys. 52 11NB07 (2013).

[12] H. Kawasaki, T. Ohshima, K. Arafune, Y. Yagyu, Y. Suda. Trans. Mater. Res. Soc. Jpn. 37147 (2012).

[13] H. Kawasaki, T. Kanazawa, S. Aoqui, T. Ohshima, Y. Yagyu, Y. Suda. Trans. Mater. Res. Soc. Jpn. 36455 (2011).

[14] T. Ohshima, S. Nakashima, T. Ueda, H. Kawasaki, Y. Suda, K. Ebihara, Thin Solid Films 506-507 106 (2006).

[15] H. Kawasaki, T. Ohshima, Y. Yagyu, T. Ihara, Y. Tanaka, Y. Suda, Trans. Mat. Res. Soc. Japan 40[2] 145 (2015).

(Received December 16, 2015; Accepted January 19, 2016) 